International Journal of Algebra, Vol. 1, 2007, no. 8, 393 - 398

\title{
Noncooperative-Equivalent Imputation Formula in Cooperative Differential Games
}

\author{
David Yeung \\ Department of Decision Sciences, Hong Kong Baptist University \\ Kowloon Tong, Hong Kong \\ and \\ Center for Game Theory, St Petersburg State University \\ Petrodivrets, 198904, St Petersburg, Russia
}

\begin{abstract}
A benchmark payoff for a player is that his imputation under cooperation must at least be equal to his noncooperative payoff at each time instant along the cooperative path. A distribution formula that yields noncooperative-equivalent imputation in cooperative differential games is presented. An exegesis of the rationale of this formula is also given.
\end{abstract}

Mathematics Subject Classification: 91A23, $91 \mathrm{~A} 12$

Keywords: Cooperative differential game, imputation, distribution formula

\section{Introduction}

Cooperative differential games represent a complex form of optimization analysis. The recent work of Yeung and Petrosyan (2004 and 2006) developed a generalized method for the derivation of analytically tractable time-consistent solutions. A benchmark payoff for a player is that his imputation under cooperation must at least be equal to his noncooperative payoff at each time instant along the cooperative path. A distribution formula that yields noncooperativeequivalent imputation in cooperative differential games is presented. An exegesis of the rationale of this formula is also given. 


\section{Mathematical Preliminaries}

Consider the $n$ - person nonzero-sum differential game with initial state $x_{0}$ and duration $T-t_{0}$. The state space of the game is $X \in R^{m}$, with permissible state trajectories $\left\{x(s), t_{0} \leq s \leq T\right\}$. The state dynamics of the game is characterized by the vector-valued differential equations:

$$
\dot{x}(s)=f\left[s, x(s), u_{1}(s), u_{2}(s), \cdots, u_{n}(s)\right], \quad x\left(t_{0}\right)=x_{0},
$$

Where $u_{i}(s) \in R^{m_{i}}$ is the control vector of player $i$.

The objective of player $i$ is

$\int_{t_{0}}^{T} g^{i}\left[s, x(s), u_{1}(s), u_{2}(s), \cdots, u_{n}(s)\right] d s+q^{i}(x(T))$, for $i \in\{1,2, \cdots, n\} \equiv N$

and $g^{i}\left[s, x(s), u_{1}(s), u_{2}(s), \cdots, u_{n}(s)\right]$ and $q^{i}(x(T))$ are non-negative. The players payoffs are transferable.

Invoking the work of Isaacs (1965) and Bellman (1957) a feedback Nash equilibrium of the game can be characterized the following well-known theorem:

Theorem 2.1 An n-tuple of strategies $\left\{\phi_{i}(t, x)\right.$, for $\left.i \in N\right\}$, provides a feedback Nash equilibrium solution to the game (1)-(2) if there exist continuously differentiable functions $V^{i}(t, x):\left[t_{0}, T\right] \times R^{m} \rightarrow R, i \in \mathbb{N}$, satisfying the following set of partial differential equations:

$$
\begin{aligned}
& -V_{t}^{i}(t, x)= \\
& \quad \max _{u_{i}}\left\{g^{i}\left[t, x, \phi(t, x), \phi_{2}(t, x), \cdots, \phi_{i-1}(t, x), u_{i}, \phi_{i+1}(t, x), \cdots, \phi_{n}(t, x)\right]\right. \\
& \left.\quad+V_{x}^{i}(t, x) f\left[t, x, \phi_{1}(t, x), \phi_{2}(t, x), \cdots, \phi_{i-1}(t, x), u_{i}, \phi_{i+1}(t, x), \cdots, \phi_{n}(t, x)\right]\right\} \\
& V^{i}(T, x)=q^{i}(x), i \in N
\end{aligned}
$$

The noncooperative payoff of player $i$ at time $t$ given that $x(t)=t$ is given by the continuously differentiable function $V^{i}(t, x)$.

Under cooperation group rationality required the players to maximize their joint payoff

$$
\int_{t_{0}}^{T} \sum_{j=1}^{n} g^{j}\left[s, x(s), u_{1}(s), u_{2}(s), \cdots, u_{n}(s)\right] d s+\sum_{j=1}^{n} q^{j}(x(T))
$$


subject to (1).

Let $\left\{\psi_{i}(s, x)\right.$, for $\left.i \in N\right\}$ denote a set of strategies leading to an optimal control solution of the problem (1) and (3) the total cooperative payoff over the interval $[t, T]$ where $t \in\left[t_{0}, T\right]$ is:

$$
\begin{aligned}
& W\left(t, x_{t}^{*}\right)= \\
& \int_{t}^{T} \sum_{j=1}^{n} g^{j}\left[s, x^{*}(s), \psi_{1}\left(s, x^{*}(s)\right), \psi_{2}\left(s, x^{*}(s)\right), \cdots, \psi_{n}\left(s, x^{*}(s)\right)\right] d s \\
& \quad+\sum_{j=1}^{n} q^{j}\left(x^{*}(T)\right) .
\end{aligned}
$$

In explicitly solvable cooperative differential games, continuously differentiable functions $W\left(t, x_{t}^{*}\right)$ and $V^{i}\left(\tau, x_{\tau}\right)$, for $i \in N$, could be obtained in closed-form.

The state dynamics under cooperation is:

$\dot{x}^{*}(s)=f\left[s, x^{*}(s), \psi_{1}\left(s, x^{*}(s)\right), \psi_{2}\left(s, x^{*}(s)\right), \cdots, \psi_{n}\left(s, x^{*}(s)\right)\right], x\left(t_{0}\right)=x_{0}$.

The corresponding optimal trajectory under cooperation can be expressed as:

$$
x^{*}(t)=x_{0}+\int_{t_{0}}^{t} f\left[s, x^{*}(s), \psi_{1}^{*}\left(s, x^{*}(s)\right), \psi_{2}^{*}\left(s, x^{*}(s)\right), \cdots, \psi_{n}^{*}\left(s, x^{*}(s)\right)\right] d s .
$$

For notational convenience, we use the terms $x^{*}(t)$ and $x_{t}^{*}$ interchangeably.

\section{Noncooperative-Equivalent Imputation For- mula}

Consider the situation when all players agree to act so that a group optimum could be obtained. A benchmark payoff for a player is that his imputation must at least be equal to his noncooperative payoff at each time instant along the cooperative path $\left\{x^{*}(t)\right\}$.

Let $\xi^{i}\left(\tau, x_{\tau}^{*}\right)$ denote the imputation to player $i$ under cooperation over the time interval $[\tau, T]$ along the cooperative path $\left\{x_{\tau}^{*}\right\}_{\tau=t_{0}}^{T}$ for $\tau \in\left[t_{0}, T\right]$. An imputation distribution procedure as in Petrosyan and Danilov (1982) and Yeung and Petrosyan (2004 and 2006) has to be formulated so that the cooperative imputation $\xi^{i}\left(\tau, x_{\tau}^{*}\right)=V^{i}\left(\tau, x_{\tau}^{*}\right)$ can be realized along the cooperative path. 
To do this we let $B_{i}\left(s, x^{*}(s)\right)$ denote the instantaneous rate of cooperative payment received by player $i$ at time $s$. In particular,

$$
\xi^{i}\left(\tau, x_{\tau}^{*}\right)=V^{i}\left(\tau, x_{\tau}^{*}\right)=\int_{\tau}^{T} B_{i}\left(s, x^{*}(s)\right) d s+q^{i}\left(x_{T}^{*}\right), \text { for } \tau \in\left[t_{0}, T\right]
$$

Theorem 3.1 A payment scheme with a terminal payment $q^{i}\left(x_{T}^{*}\right)$ at time $T$ and an instantaneous rate of payment at time $\tau \in\left[t_{0}, T\right]$ along the cooperative trajectory $\left\{x_{\tau}^{*}\right\}_{\tau=t_{0}}^{T}$ being

$$
\begin{aligned}
B_{i}\left(\tau, x_{\tau}^{*}\right)= & -V_{\tau}^{i}\left(\tau, x_{\tau}^{*}\right) \\
& -V_{x_{\tau}^{*}}^{i}\left(\tau, x_{\tau}^{*}\right) f\left[\tau, x_{\tau}^{*}, \psi_{1}\left(\tau, x_{\tau}^{*}\right), \psi_{2}\left(\tau, x_{\tau}^{*}\right), \cdots, \psi_{n}\left(\tau, x_{\tau}^{*}\right)\right]
\end{aligned}
$$

yield the noncooperative-equivalent imputation

$$
\xi^{i}\left(\tau, x_{\tau}^{*}\right)=\int_{\tau}^{T} B_{i}\left(s, x^{*}(s)\right) d s+q^{i}\left(x_{T}^{*}\right)=V^{i}\left(\tau, x_{\tau}^{*}\right), \text { for } \tau \in\left[t_{0}, T\right]
$$

\section{Proof.}

Using (7) one can obtain the identity:

$$
\int_{t_{0}}^{\tau} B_{i}\left(s, x^{*}(s)\right) d s+V^{i}\left(\tau, x_{\tau}^{*}\right) \equiv V^{i}\left(t_{0}, x_{0}\right) \text {. for } \tau \in\left[t_{0}, T\right]
$$

Differentiating (8) with respect to $\tau$ yields

$$
B_{i}\left(\tau, x_{\tau}^{*}\right)=-d V^{i}\left(\tau, x_{\tau}^{*}\right) / d \tau=-V_{\tau}^{i}\left(\tau, x_{\tau}^{*}\right)-V_{x_{\tau}^{*}}^{i}\left(\tau, x_{\tau}^{*}\right) \dot{x}^{*}(\tau)
$$

Invoking (5) we obtain

$B_{i}\left(\tau, x_{\tau}^{*}\right)=-V_{\tau}^{i}\left(\tau, x_{\tau}^{*}\right)-V_{x_{\tau}^{*}}^{i}\left(\tau, x_{\tau}^{*}\right) f\left[\tau, x_{\tau}^{*}, \psi_{1}\left(\tau, x_{\tau}^{*}\right), \psi_{2}\left(\tau, x_{\tau}^{*}\right), \cdots, \psi_{n}\left(\tau, x_{\tau}^{*}\right)\right]$

Hence Theorem 3.1 follows.

Theorem 3.1 yields a distribution formula for noncooperative-equivalent imputation in a cooperative differential game with dynamics (1) and payoffs (2). Such a formula can be obtained in closed form for any explicitly solvable games. 


\section{An Exegesis of the formula}

In this section an exegesis of the ratioale for the noncooperative-equivalent imputation formula (11) is presented. Note that the Isaacs-Bellman equation in Theorem 2.1 for a feedback Nash equilibrium in the noncooperative game (1) and (2) leads to

$$
\begin{aligned}
-V_{\tau}^{i}\left(\tau, x_{\tau}^{*}\right)= & g^{i}\left[\tau, x_{\tau}^{*}, \phi_{1}\left(\tau, x_{\tau}^{*}\right), \phi_{2}\left(\tau, x_{\tau}^{*}\right), \cdots, \phi_{n}\left(\tau, x_{\tau}^{*}\right)\right] \\
& +V_{x_{\tau}^{*}}^{i}\left(\tau, x_{\tau}^{*}\right) f\left[\tau, x_{\tau}^{*}, \phi_{1}\left(\tau, x_{\tau}^{*}\right), \phi_{2}\left(\tau, x_{\tau}^{*}\right), \cdots, \phi_{n}\left(\tau, x_{\tau}^{*}\right)\right] \\
& \text { for } \tau \in\left[t_{0}, T\right]
\end{aligned}
$$

Using (12) the distribution formula in (10) can be expressed as:

$$
\begin{aligned}
B_{i}\left(\tau, x_{\tau}^{*}\right)= & g^{i}\left[\tau, x_{\tau}^{*}, \phi_{1}\left(\tau, x_{\tau}^{*}\right), \phi_{2}\left(\tau, x_{\tau}^{*}\right), \cdots, \phi_{n}\left(\tau, x_{\tau}^{*}\right)\right] \\
& +V_{x_{\tau}^{*}}^{i}\left(\tau, x_{\tau}^{*}\right)\left\{f\left[\tau, x_{\tau}^{*}, \phi_{1}\left(\tau, x_{\tau}^{*}\right), \phi_{2}\left(\tau, x_{\tau}^{*}\right), \cdots, \phi_{n}\left(\tau, x_{\tau}^{*}\right)\right]\right. \\
& \left.-f\left[\tau, x_{\tau}^{*}, \psi_{1}\left(\tau, x_{\tau}^{*}\right), \psi_{2}\left(\tau, x_{\tau}^{*}\right), \cdots, \psi_{n}\left(\tau, x_{\tau}^{*}\right)\right]\right\}
\end{aligned}
$$

However, along the cooperative path $\left\{x_{\tau}^{*}\right\}_{\tau=t_{0}}^{T}$, the instantaneous rate of payoff to player $i$ is :

$$
g^{i}\left[\tau, x_{\tau}^{*}, \psi_{1}\left(\tau, x_{\tau}^{*}\right), \psi_{2}\left(\tau, x_{\tau}^{*}\right), \cdots, \psi_{n}\left(\tau, x_{\tau}^{*}\right)\right] \text { at time instant } \tau .
$$

In order for player $i$ to realize an instantaneous rate of payoff equaling $B_{i}\left(\tau, x_{\tau}^{*}\right)$ a noncooperative-equivalent compensation formula can be obtained as

$$
\begin{aligned}
\vartheta_{i}\left(\tau, x_{\tau}^{*}\right)= & B_{i}\left(\tau, x_{\tau}^{*}\right)-g^{i}\left[\tau, x_{\tau}^{*}, \psi_{1}\left(\tau, x_{\tau}^{*}\right), \psi_{2}\left(\tau, x_{\tau}^{*}\right), \cdots, \psi_{n}\left(\tau, x_{\tau}^{*}\right)\right] \\
= & g^{i}\left[\tau, x_{\tau}^{*}, \psi_{1}\left(\tau, x_{\tau}^{*}\right), \psi_{2}\left(\tau, x_{\tau}^{*}\right), \cdots, \psi_{n}\left(\tau, x_{\tau}^{*}\right)\right] \\
& -g^{i}\left[\tau, x_{\tau}^{*}, \phi_{1}\left(\tau, x_{\tau}^{*}\right), \phi_{2}\left(\tau, x_{\tau}^{*}\right), \cdots, \phi_{n}\left(\tau, x_{\tau}^{*}\right)\right] \\
& +V_{x_{\tau}^{*}}^{i}\left(\tau, x_{\tau}^{*}\right)\left\{f\left[\tau, x_{\tau}^{*}, \phi_{1}\left(\tau, x_{\tau}^{*}\right), \phi_{2}\left(\tau, x_{\tau}^{*}\right), \cdots, \phi_{n}\left(\tau, x_{\tau}^{*}\right)\right]\right. \\
& \left.-f\left[\tau, x_{\tau}^{*}, \psi_{1}\left(\tau, x_{\tau}^{*}\right), \psi_{2}\left(\tau, x_{\tau}^{*}\right), \cdots, \psi_{n}\left(\tau, x_{\tau}^{*}\right)\right]\right\}
\end{aligned}
$$

In formula (15) the term

$$
\begin{array}{r}
g^{i}\left[\tau, x_{\tau}^{*}, \phi_{1}\left(\tau, x_{\tau}^{*}\right), \phi_{2}\left(\tau, x_{\tau}^{*}\right), \cdots, \phi_{n}\left(\tau, x_{\tau}^{*}\right)\right] \\
-g^{i}\left[\tau, x_{\tau}^{*}, \psi_{1}\left(\tau, x_{\tau}^{*}\right), \psi_{2}\left(\tau, x_{\tau}^{*}\right), \cdots, \psi_{n}\left(\tau, x_{\tau}^{*}\right)\right]
\end{array}
$$

yields the difference between player $i^{\prime}$ s rate of instantaneous payoffs when he uses the noncooperative strategy and that when he adopts the cooperative 
strategy. The term $V_{x_{\tau}^{*}}^{i}\left(\tau, x_{\tau}^{*}\right)$ reflects the marginal effects of a change in the state variables on agent $i$ ' noncooperative payoff. The term $f\left[\tau, x_{\tau}^{*}, \phi_{1}\left(\tau, x_{\tau}^{*}\right)\right.$, $\left.\phi_{2}\left(\tau, x_{\tau}^{*}\right), \cdots, \phi_{n}\left(\tau, x_{\tau}^{*}\right)\right]$ yields the instantaneous change of the states over time if the agents act noncooperatively, while the term $f\left[\tau, x_{\tau}^{*}, \psi_{1}\left(\tau, x_{\tau}^{*}\right)\right.$, $\psi_{2}\left(\tau, x_{\tau}^{*}\right), \cdots, \psi_{n}\left(\tau, x_{\tau}^{*}\right)$ ] yields the instantaneous change of the states over time if the agents act cooperatively.

Hence, the expression

$$
\begin{array}{r}
V_{x_{\tau}^{*}}^{i}\left(\tau, x_{\tau}^{*}\right)\left\{f\left[\tau, x_{\tau}^{*}, \phi_{1}\left(\tau, x_{\tau}^{*}\right), \phi_{2}\left(\tau, x_{\tau}^{*}\right), \cdots, \phi_{n}\left(\tau, x_{\tau}^{*}\right)\right]\right. \\
\left.-f\left[\tau, x_{\tau}^{*}, \psi_{1}\left(\tau, x_{\tau}^{*}\right), \psi_{2}\left(\tau, x_{\tau}^{*}\right), \cdots, \psi_{n}\left(\tau, x_{\tau}^{*}\right)\right]\right\}
\end{array}
$$

represents the compensation to player $i$ when the change in the state variable follows the cooperative trajectory instead of the noncooperative path. To sum up, at time instant $\tau$ the compensation to player $i$ leading to the noncooperative-equivalent instantaneous rate of payoff $B_{i}\left(\tau, x_{\tau}^{*}\right)$ consists of (i) the compensation on the difference between player $i^{\prime} \mathrm{s}$ rate of instantaneous payoffs when he uses the noncooperative strategy and that when he adopts the cooperative strategy, and (ii) the compensation to agent $i$ for the difference in the change in the state variable on the cooperative trajectory and that on the noncooperative path.

ACKNOWLEDGEMENTS: Financial support from the Hong Kong Baptist University (Grants FRG/05-06/II-22 and FRG/06-07/II-39 ) is gratefully acknowledged.

\section{References}

[1] R. Bellman, Dynamic programming, Princeton University Press, Princeton, NJ, 1957.

[2] R. Isaacs, Differential Games, Wiley, New York, 1965.

[3] L. A Petrosyan and N. N. Danilov, Cooperativbe differential games and their applications, Tomskogo University, Tomsk, 1982.

[4] D. W. K. Yeung and L. A. Petrosyan, Subgame Consistent Cooperative Solutions in Stochastic Differential Games, Journal of Optimization Theory and Applications, 120 (2004), 651-666.

[5] D. W. K. Yeung and L. A. Petrosyan, Cooperative Stochastic Differential Games, Springer-Verlag, New York, 2006.

Received: March 15, 2007 